\title{
TTR
}

Traduction, terminologie, re?daction

\section{La traduction citoyenne n'est pas une métaphore}

\section{Salah Basalamah}

Volume 18, numéro 2, 2e semestre 2005

Traduction engagée

Translation and Social Activism

URI : https://id.erudit.org/iderudit/015746ar

DOI : https://doi.org/10.7202/015746ar

Aller au sommaire du numéro

\section{Éditeur(s)}

Association canadienne de traductologie

ISSN

0835-8443 (imprimé)

1708-2188 (numérique)

Découvrir la revue

Citer cet article

Basalamah, S. (2005). La traduction citoyenne n'est pas une métaphore. TTR, 18(2), 49-69. https://doi.org/10.7202/015746ar

\section{Résumé de l'article}

Après une mise en question de l'étroitesse de la définition de la traduction comme une opération de transfert exclusivement interlinguistique, cet article explore la possibilité d'élargir les frontières définitoires, établies au sein de la discipline traductologique, par un retour sur la valeur, pour le moins dépréciée, de la traduction métaphorique. La tentative consiste à faire, d’une part, un détour par l'éthique du traducteur qui impose sa visibilité et, par conséquent, sa participation citoyenne dans l'effort de rapprochement et d'intercompréhension des différences, et, d'autre part, une resémantisation du caractère métaphorique de la traduction dès lors qu'elle est porteuse d'enjeux aussi réels et circonstanciés que ceux, par exemple, de la difficile intégration des musulmans occidentaux et de l'expression de leur religiosité dans les sociétés laïques. En somme, la traduction métaphorique ainsi comprise nous conduit à considérer le nouveau concept de " traduction citoyenne ".

Mots-clés : métaphore, définition de la traduction, citoyenneté, visibilité, démocratie.

Tous droits réservés ( C TTR: traduction, terminologie, rédaction — Les auteurs, Ce document est protégé par la loi sur le droit d’auteur. L’utilisation des 2007 services d'Érudit (y compris la reproduction) est assujettie à sa politique d'utilisation que vous pouvez consulter en ligne.

https://apropos.erudit.org/fr/usagers/politique-dutilisation/ 


\section{La traduction citoyenne n'est pas une métaphore}

\section{Salah Basalamah}

Nombreux, hier et aujourd'hui, sont ceux qui admettent que la traduction, de même que l'interprétation (Steiner, 1998; Barsky, 2000, p. 46), ne se réduisent pas au transfert d'une langue à une autre, mais qu'elles vont bien au-delà. En effet, depuis les «Aspects linguistiques de la traduction » de Jakobson (1963), où « il s'inspire explicitement des écrits de Charles S. Peirce » (Oustinoff, 2003, p. 21), une nouvelle dimension de la traduction semble avoir été suggérée : le passage d'une forme d'expression à une autre, ou entre deux systèmes de signes distincts, constitue également de la traduction. Bien que selon une perspective plus rigoriste on parlerait aujourd'hui plutôt d'adaptation pour les œuvres qui passent d'un genre littéraire à un autre, il n'en reste pas moins que la «traduction intersémiotique » jakobsonienne est une tentative de décrire plus radicalement un phénomène qui dépasse les frontières du seul langage, au sens le plus strict.

En ce sens, la culture n'est plus seulement l'un des facteurs les plus déterminants dans la traduction d'un texte étranger, mais bien plus l'objet même du transfert. En effet, s'il est une chose que le « tournant culturel » nous a appris (Snell-Hornby, 1990; Bassnett et Lefevere, 1990), c'est que la traduction ne peut plus se cantonner dans la tautologique «traduction comme texte », mais bien plutôt s'élargir vers l’horizon de la « traduction des cultures ». Bien plus, depuis les années 1950 (Lienhardt, 1967), l’anthropologie sociale avait déjà le souci de penser le problème de la traduction des autres cultures, en vue de les comprendre, comme des systèmes de pensée pour lesquels il faut trouver des équivalents dans sa propre culture. En convertissant des configurations culturelles et symboliques différentes vers d'autres, 
traducteur et ethnographe auraient donc des tâches similaires (Valero-Garcès, 1995; Laplantine, 1995; Wolf, 2002).

L'autre leçon dont on peut légitimement revendiquer l'héritage, c'est bien celle des auteurs poststructuralistes qui, loin de sombrer dans le nihilisme absolu, nous invitent plutôt à prendre acte de la naissance d'un nouvel acteur fondamental dans la production du sens : le lecteur. De tous ceux qui peuvent s'identifier à la fonction de réception active, le traducteur est certes l'un des plus attentifs, mais également l'un des plus hésitants à assumer sa subjectivité. Les dimensions culturelle et sociale de la traduction n'ont pourtant jamais été aussi évidentes dans la fragilisation de principes aussi profondément ancrés que l'invisibilité du sujet traducteur.

Or, l'étroitesse d'une conception strictement instrumentale de la traduction peut s'exprimer de différentes manières, selon l'institution concernée. Robert Barsky, dans son ouvrage Arguing and Justifying (2000), s'insurge, quant à lui, contre la limitation, par les autorités gouvernementales responsables de filtrer les requérants d'asile aux frontières, du rôle de l'interprète et affirme :

[...] indeed, the interpreter's role as intermediary is generally downplayed and restricted to basic translation tasks. This occurs despite the fact that discourse analysis research specifically points out the limitations of such an approach... (Barsky, 2000, p. 57)

Il continue plus loin :

There is, in fact, ample evidence in domains including linguistics, anthropology, sociology, and law that translation is anything but straightforward. (id.)

Dans le même sens, il donne également l'exemple de l'étude d'Adda Bozeman (1971) sur le mouvement de traduction du bouddhisme entre l'Inde et la Chine entre 150 et 120 avant J.-C. ${ }^{1}$ Opération difficile,

\footnotetext{
${ }^{1}$ In 148 C.E. a monk named An Shih-kao, from the Central Asian kingdom of Kusha, began translating Indian Buddhist texts into Chinese in Lo-yang, which was to become the capital of the later Han dynasty. An Shih-kao and a number of other monks (mostly from Central Asia) translated about thirty Buddhist texts during the next three decades. The early translators used a translation system termed "matching concepts" (ko-i), which was to have important ramifications for the development of Chinese Buddhism. Realizing that China had a highly developed culture and that Chinese tended to view people from other countries
} 
s’il en est, en raison de la disparité du sanskrit et du chinois, du bouddhisme lui-même et du taoïsme :

So what was required? Not a simple translation, "but rather an interpretation of the images and experiences that had originally inspired the words; and this meant in the final analysis that all of India and all of China had to be understood before the separate aspects of Buddhism could be made meaningful to the Chinese » [Bozeman 1971, pp. 4-5].

This brings us back to the requirement of situating the discourse into a broader social discourse universe as a means of understanding the utterances themselves, but adds to it the task of positioning these utterances into a larger socio-historical framework for adequate interpretation. (Barsky, 2001, p. 58) ${ }^{2}$

C'est dire que non seulement la tâche du traducteur/interprète ne peut être conçue en faisant abstraction du cadre plus élargi de son opération, tant du point de vue spatial que temporel, mais on ne saurait non plus faire l'économie du rôle implicite de la subjectivité, de son agency au cœur de cette transaction. D'une part, traduire ne se limite pas à un texte ou un discours, mais à tout le contexte qui les forme et les informe; et d'autre part, l'historicité du traducteur le situe et affirme sa subjectivité au point d'être critique :

[...interpreters] should be legally recognized as critical intermediaries [...] rather than as innocuous translating devices. [...] they should have the latitude to assist the claimant by intervening with questions and clarifications that are both pertinent to the case and liable to assist the claimant. (id.) $)^{3}$

Ainsi, Barsky ne se satisfait pas de la banale (et pourtant rare) compétence du traducteur, puisque l'intervention de l'interprète - en

as uncouth barbarians, the early translators used indigenous terminology — particularly Taoist terminology — to translate Sanskrit technical terms. One result of this practice was that it made many foreign ideas more palatable to Chinese readers, but it also inevitably colored the translations to such an extent that for the first few centuries after Buddhism's arrival in China, many Chinese believed it to be another version of Taoism. (http://www.twilightbridge.com/hobbies/festivals/buddha/spread.htm)

\footnotetext{
${ }^{2}$ C’est nous qui soulignons.

${ }^{3}$ C'est nous qui soulignons.
} 
situation d'intermédiaire entre le représentant de l’État et le réfugié doit également revêtir un caractère proactif, ménager les avenues d'un dialogue constructif, voire se montrer solidaire.

Or, si la traduction et l'interprétation ne doivent pas être considérées comme des activités dénuées de subjectivité et de contextualisation, comment se fait-il que le champ de la traductologie paraisse si étroit quand il s'agit de la traduction comme action solidaire?

\section{La traductologie institutionnalisée : l'étroitesse du champ des possibles}

Bien que nouvelle venue dans un nombre croissant de départements de linguistique, de littératures et/ou de traduction, la traductologie s'est enfin imposée dans le monde universitaire comme une branche de moins en moins contestée des "nouvelles sciences humaines ». Mais un tel établissement au carrefour des disciplines dont la langue et la culture sont les principaux objets d'étude ne peut se faire qu'au prix d'une «négociation territoriale ». En effet, à voir mesurer certaines conséquences de l'institutionnalisation de la traductologie, on se rend compte que le piège dans lequel sont tombées nombre de disciplines plus anciennes est bien menaçant. Après la parcellarisation des connaissances et l'enfermement disciplinaire constatés dans les sciences humaines classiques (Gusdorf, 1986) par l'affirmation abusive de leurs frontières respectives en vue de protéger leurs « spécificités » et de marquer ainsi d'un limes méthodologique le champ de leur matière (Morin, 1999), on commence à voir poindre à l'horizon de la traductologie les mêmes dangers.

Pourtant, la traductologie, nous a-t-on répété à satiété - alors que nous étions étudiants - est un domaine d'étude inter- et pluridisciplinaire par excellence. C'est là tout son intérêt et sa promesse. Associée à toutes les branches des sciences humaines et même de certaines sciences médicales et domaines technologiques, la traductologie a démontré à profusion, dans sa recherche fondamentale et appliquée, la variété d'horizons auxquels elle est capable de s'ouvrir (Nouss, 1995, p. 341).

La raison en est certainement qu'au cœur de ce nouveau champ disciplinaire se trouve la traduction, symbole même de l'hétérogénéité et du rapport à l'altérité. Or, tout le défi pour la traductologie consiste donc 
justement - dans son effort de convoquer les autres savoirs, de s'y frotter et par conséquent de s'excentrer de son noyau dur - à consacrer une bonne part de son énergie à élargir constamment le sens de la traduction au double risque, il est vrai, si elle ne le fait pas, de s'aplatir (au propre et au figuré), de s'aliéner totalement par une sorte d'assimilation en abîme et de ne devenir en somme que l'instrument des autres.

Cependant, il apparaît malheureusement que dans les zones les plus institutionnalisées de la traductologie (universités, associations professionnelles, maisons d'édition à diffusion universitaire, etc.), le sens de la traduction tende à s'isoler et à se confiner dans une exclusivité quelque peu masochiste. Ainsi, contrairement aux bonnes paroles des philosophes aux intérêts métissés, aux théoriciens babéliens et aux activistes interculturels hyperhybridés, il reste que, aux yeux d'aucuns, la signification de la traduction ne peut, malgré tout, " faire l'économie » du processus de transfert linguistique que l'on décrit pourtant, et en même temps, comme une frontière limitative et contraignante : "Mais non, voyons! traduire c'est bien plus que cela... »

Soyons francs. De quoi a-t-on peur au juste ? Oui, bien entendu, les jeux de pouvoir des institutions et de leurs différents acteurs peuvent constituer une source non négligeable de pression. Mais en réalité, faut-il le souligner, l'enjeu est tout autant politique qu'intellectuel et les conséquences du cloisonnement des disciplines ne peuvent qu'être néfastes :

La frontière disciplinaire, son langage et ses concepts propres isolent la discipline par rapport aux autres et par rapport aux problèmes qui chevauchent les disciplines. L'esprit hyperdisciplinaire risque alors de se former, comme un esprit de propriétaire qui interdit toute circulation étrangère dans sa parcelle de savoir. (Morin, 1990, p. 1)

Autrement que la traduction de la métaphore (qui fait l'objet du plus grand nombre de recherches), c'est la traduction comme métaphore que l'on craint. Bien que développée par plus d'un traductologue (notamment les théoriciens postcoloniaux, les ethnographes et les anthropologues), elle reste plutôt marginale. Sa seule vertu serait pédagogique: elle permet d'imaginer les différents niveaux de déplacement que recouvre la traduction, au-delà de la seule communication du sens. C'est que la traduction, élevée au rang de paradigme de l'interrelation des différences et de la fécondation mutuelle des visions du monde et des signes afférents à celles-ci, suppose sa méta-phorisation : il s'agit, par le dépassement du champ 
rigoureusement textologique (sans toutefois s'en départir), de conserver en même temps son ancrage au sein du réel social. La traduction des concepts d'une discipline, des sensibilités religieuses d'une communauté, des schémas mentaux d'un groupe ethnique ou social, de l'intelligence politique d'un peuple ou encore de l'éthique économique d'une culture ne peut être considérée comme une activité étrangère à l'action proprement traductive de transformer des significations dans des sphères de compréhension différentes. Assumant la validité du champ de la représentation comme seul horizon, la traduction des «cultures comme textes » n'en est pas moins l'un des axes bien établis de la très sérieuse et confirmée réflexion anthropologique (Clifford et Marcus, 1986).

Alors qu'il faudrait entreprendre toute une étude pour réhabiliter le principe de la traduction comme métaphore, ${ }^{4}$ il nous suffira pour l'instant de nous appuyer sur les acquis des rares auteurs qui en ont traité ou l'ont admise comme valide dans le champ de la traductologie (Rafael, 1988; Copeland, 1991; Cheyfitz, 1991; Niranjana, 1992).

Cela dit, avant d'aller plus loin, notons tout de même que dans le différend qui nous occupe ici avec les tenants inavoués de la définition stricte de la traduction, on voit se reproduire la même réserve que celle exprimée - par la majorité - à l'endroit de l'action, non pas conçue dans sa généralité, mais prise au sens plus spécifique de l'engagement et de l'activisme social. Avec cette dernière expression, force est de constater que nous sommes désormais parvenus au cœur du politique : " Attention, terrain miné ! », diraient certains. De fait, la traduction, si on lui concède tout au plus le rôle de médiatrice entre les cultures, en plus des langues, n'en doit pas moins garder le texte pour point d'ancrage premier et lieu d'opération privilégié. La textualité serait ainsi pour certains traductologues le critère de validité ultime. Toute dérive, voire toute tentative de sortir du texte équivaudrait alors tout bonnement à quitter le terrain de la traductologie. La traduction est donc avant tout un phénomène qui relève de la parole; c'est pourquoi, l'action n'aurait de pertinence que de manière "métaphorique », autrement dit, lointaine, trop construite.

En ce sens, la réflexion de Paul Ricœur nous paraît utile pour en faire la critique. Dans son ouvrage Du texte à l'action, Ricœur montre la convergence, voire l'interdépendance du texte et de l'action :

\footnotetext{
${ }^{4}$ Objet probable d'un futur ouvrage.
} 
Je dirai en bref que d'un côté la notion de texte est un bon paradigme pour l'action humaine, de l'autre l'action est un bon référent pour toute une catégorie de textes. En ce qui concerne le premier point, l'action humaine est à bien des égards un quasi-texte. Elle est extériorisée d'une manière comparable à la fixation caractéristique de l'écriture. En se détachant de son agent, l'action acquiert une autonomie semblable à l'autonomie sémantique d'un texte; elle laisse une trace, une marque; elle s'inscrit dans le cours des choses et devient archive et document. À la manière d'un texte, dont la signification s'arrache aux conditions initiales de sa production, l'action humaine a un poids qui ne se réduit pas à son importance dans la situation initiale de son apparition, mais permet la réinscription de son sens dans de nouveaux contextes. Finalement, l'action, comme un texte, est une œuvre ouverte, adressée à une suite infinie de « lecteurs » possibles. (Ricœur, 1986, p. 195)

C'est donc dire qu'à la textualité traductive doit correspondre un activisme traductif. Sans définir lequel des deux espaces est l'original de l'autre, le texte et l'action sont, en revanche, chacun à leur tour, la représentation de l'autre. La répétition de l'action devient alors possible, comme celle du texte infiniment régénéré par l'émergence d'une nouvelle historicité, d'une nouvelle parole. La traduction de l'un à l'autre est ainsi une sorte de métaphore du déplacement qui se produit à chaque fois que le texte s'ouvre sur la nécessaire complémentarité du monde et que l'action appelle le texte comme un miroir pour se réinventer. Le " livre du monde », texte de référence par excellence, est un signe qui ne prend sens que dans sa corrélation avec la dynamique de son perpétuel avènement.

\section{Traduction métaphorique : projet d'une redéfinition}

Mais si traduction et métaphore sont aussi convergentes et interdépendantes que sont le texte et l'action, qu'est-ce alors qu'une traduction métaphorique si elle n'agit que dans le cercle fermé de la seule textualité ? Tel serait le paradoxe de la traduction conçue exclusivement comme transfert linguistique. Or, ce que nous proposons de nommer «traduction citoyenne " (et qui n'est qu'apparemment métaphorique) est justement le lieu même où se concentre toute la densité du réel. C’est parce que la traduction a été déplacée hors de sa niche confortable de la textualité qu'elle peut prétendre à la réforme sociale, ou en termes plus poétiques, à « changer la vie ». 
Que ce soit là pour nous un postulat: "la traduction métaphorique doit faire à la fois l'objet d'une resémantisation et d'une réhabilitation ». Resémantisation parce que son exclusion hors du champ de la traductologie l'a non seulement dévalorisée, mais elle l'a également évidée de toute signification pertinente à la traduction; et réhabilitation parce qu'il existe des phénomènes de société contemporains où sa valeur opératoire est encore manifestement insoupçonnée (les nombreux espaces de conflit et par là même de non-traduction). Avant de nous y attarder plus longuement dans une recherche ultérieure, posons pour l'instant ce premier jalon en vue d'appuyer notre propos.

On nous a assez rappelé que le latin translatio et le grec metaphora signifient tous deux et tout à la fois métaphore et traduction pour y chercher non seulement une communauté de destin, mais surtout une portée extralinguistique à la traduction. C'est le phénomène médiéval de translatio et imperii et studii qui en est la première manifestation politique (Copeland, 1991). Si Cheyfitz et Rafael affirment que la mission impérialiste de la traduction était de « traduire l'autre dans les termes de l'empire » (1991) et de le « convertir » en une représentation de soi (1988), il n'est pas certain que la métaphorisation de ce type d'acte traductif ne soit plus valide à l'heure où l'extension irrévocable de la domination des plus faibles passe plus que jamais par le chemin de la médiatisation absolue ou, en d'autres termes, la traduction systématiquement occidentalocentrique de toute altérité (Venuti, 1998, pp. 158-189).

Mais n'est-il pas temps de ne plus concevoir le processus traductif selon le schéma triadique traditionnel qui met aux prises l'interlocuteur de départ, le traducteur et l'interlocuteur d'arrivée ? L'autre, celui que l'on traduit et que l'on ramène à soi, est-il donc nécessairement cette altérité radicale et étrangère vouée à l'oblitération dans des sociétés (notamment occidentales) pourtant hypermétissées ? L'autre n'est-il pas également, voire souvent aujourd'hui, cette différence dont le visage récurrent nous impose une présence quotidienne, voire concitoyenne - mais non reconnue comme telle dans les faits ? La différence, toujours traitée comme altérité, n’est depuis longtemps plus «à nos portes ", mais au cœur même de nos espaces sociaux les plus intimes. Peut-être est-ce justement là l'une des raisons du caractère si affectif des réactions xénophobes de certains groupes dans les sociétés les plus urbanisées du globe. 
Deux constats. Premièrement, il est trivial d'affirmer qu'il n'existe pas de traduction neutre. On dira avec Jean Peeters que le traducteur est nécessairement un «traducteur d'arrivée » ou « ethnocentrique », selon la terminologie de Berman :

Pour interculturelle que soit la traduction, elle n'en reste donc pas moins une production historiquement donnée de la langue d’arrivée s'adressant à des interlocuteurs-consommateurs dans la langue d'arrivée, et non pas à des interlocuteurs dans la langue de départ. Pour altruiste que soit la traduction, elle se doit de mettre l'Étranger à la portée des interlocuteurs de la langue d'arrivée; il doit être consommable. [...] L’interlocution médiatisée par le métier de la traduction n'échappe pas à l'ethnocentrisme de la langue d'arrivée et ne peut s'inscrire que par rapport à ce point de repère sociolinguistique d’où est envisagée la langue de départ. (1999, p. 273)

Deuxièmement, notre conception de la position du traducteur diffère de celle notamment de Barsky puisqu'elle n'est pas celle d'un tiers médiateur. Ce dernier serait en revanche, et à la manière du Blendlinge d'Anthony Pym (1997), un médiateur qui peut être lui-même partie prenante du processus de traduction en étant à la fois traduit et traducteur. Le sujet traducteur retrouve toute son épaisseur et ne saurait se départir de sa tâche dans la mesure où c'est son identité même qui est en jeu. De fait, puisqu'il n'est plus question de neutralité (que ce soit dans les faits ou selon l'éthique que développe Barsky en faveur des réfugiés) et que l'équation traductive est restreinte à deux termes au lieu de trois, peut-être faudrait-il désormais concevoir la traduction, non plus dans son rapport avec l'étranger radical, l'étranger de l'extérieur, mais avec celui qui parle la même langue, l'immigrant et ses descendants (éternellement "issus de l'immigration ») ou celui qu'on appellerait plus abruptement « le loup dans la bergerie »...

Le paradoxe d'une telle situation de traduction serait celui de traduire de manière ethnocentrique celui que l'on persiste à identifier comme étranger au sein de ses propres murs mais dont la langue et la culture sont pourtant communes. La différence entre un citoyen «autochtone » et un autre immigrant ou d'origine immigrante consisterait dans les faits en la banale pluralité des identités dont il se réclame. Et pourtant, qui aujourd'hui ne se prévaut d'une telle revendication? C'est que la différence a des limites que les représentations, par exemple orientalistes, ne sauraient parfois franchir : on va du « comment peut-on être musulman(e) et féministe à la fois ? » 
au plus radical « comment peut-on être musulman en 2006 ? » à défaut de ne plus être le Persan de Montesquieu...

À bien examiner ce nouveau type de scénario traductif, l'on est bien forcé d'admettre qu'une traductologie qui n'intègre ni le hors-texte ni le voisin de pallier étranger avec lequel on partage langue et culture est une traductologie qui ne sait pas prendre langue avec le monde qui l'entoure.

\section{Enjeux de l'islam occidental}

Pour illustrer ce qui précède, je me propose d'introduire mon expérience personnelle en tant que musulman occidental ${ }^{5}$ et de témoigner de ce que je perçois de là où je suis, en l'occurrence à la fois de l'intérieur et de l'extérieur, comme traduit et traducteur...

En effet, alors que le monde musulman est affecté par les maux les plus divers (de l'analphabétisme à la corruption, du terrorisme aux catastrophes naturelles et du sous-développement à la colonisation militaire - entre mille autres choses) et que les médias s'en font l'écho le plus spectaculaire, on ne peut manquer de constater que les défis rencontrés par les nouvelles générations des musulmans occidentaux au sein de leurs propres sociétés se prêtent particulièrement à notre réflexion traductologique. Quelques constats tout d'abord du point de vue musulman.

Malgré la communauté de langue, d'éducation, de culture et de citoyenneté, les musulmans occidentaux font face à une résistance notable à leur intégration dans le champ du politique, si ce n'est comme représentants et symboles délibérés de la "diversité culturelle » portée comme slogan-prétexte pour agir contre le péché d'homogénéité dont on ne veut désormais plus être accusé. Aujourd'hui, la méfiance et le soupçon qui entourent les musulmans occidentaux comme véritables ressortissants de leurs pays, désormais de fait, ressemblent étrangement à la méfiance et au soupçon qui ont entouré les juifs européens dans les siècles précédents jusqu'à l'ignominie de l'holocauste (double appartenance, double langage, double allégeance, etc.). La multiplicité identitaire serait en fait une « duplicité », d'une infidélité probable si ce n'est tout à fait avérée pour certains.

\footnotetext{
${ }^{5}$ En me basant sur mes expériences européennes passées (suisse et française en particulier) et plus récemment québécoise.
} 
Alors que les premières générations étaient des populations décrites à juste titre comme étant « immigrantes ", il est à noter que les générations suivantes, jusqu'aux plus récentes sont toujours présentées comme immigrantes ou de descendance immigrante. La laïcité est un espace qui définit l'équidistance entre l'État et toutes les religions en présence. Cependant, la dernière loi française sur les signes religieux (15 mars 2004) et la motion de l'Assemblée nationale québécoise pour empêcher les musulmans de créer des tribunaux d'arbitrage ${ }^{6}$ (26 mai 2005) sont discriminatoires et non conformes aux principes de justice et d'égalité entre les citoyens, de quelque appartenance confessionnelle qu'ils soient.

En revanche, du point de vue du courant majoritaire de la société, ${ }^{7}$ la perception est autrement différente. Il n’y a pas le moindre doute: les musulmanes sont nécessairement opprimées puisqu'elles portent le foulard. C'est d'ailleurs là le symbole même de la soumission. La violence est une caractéristique intrinsèque de l'islam qui se vérifie dans les actions des musulmans. La religion musulmane serait mieux accueillie si elle pouvait avoir le même type de présence sociale que le christianisme. Toute expression sociale du religieux musulman est une intrusion du privé dans la sphère publique ainsi qu'une atteinte aux principes de la laïcité et de l'État de droit. L'intégration des musulmans occidentaux est souvent conçue dans la doxa comme un devoir d'assimilation unilatéral incombant surtout aux concernés et si peu à la «société d'accueil ». Une citoyenneté modèle serait en somme une citoyenneté sans islam, sinon avec moins d'islam, etc.

Au regard de ces visions apparemment antagonistes, on serait en droit de se demander comment les citoyens d'une même société pourraient envisager de vivre ensemble en harmonie ? Est-ce donc là une telle utopie que toute tentative de compréhension mutuelle serait vouée à l'échec ? La traduction a-t-elle les moyens de contribuer à une meilleure compréhension des disparités de perceptions d'un phénomène aussi problématique que la place du religieux dans la société laïque de l'Occident postmoderne et pluriel, ou n'a-t-elle ni la vocation ni la prérogative d'intervenir dans un dossier aussi grave ? Nous avons

\footnotetext{
${ }^{6}$ Alors que nul ne l’a réclamé ni ne peut constitutionnellement le faire.

${ }^{7}$ Au sens où il est répercuté par la plupart des médias.
} 
pourtant la prétention et l'espoir qu'il n'en est rien, même si les difficultés sont aussi énormes qu'elles paraissent.

\title{
Traduction citoyenne : définition d'un champ
}

Pour atténuer de telles tensions sociales, nous pensons qu'il est nécessaire de concevoir un traducteur ${ }^{8}$ et une traduction d'un type nouveau. À partir des résultats d'une précédente étude qui mettait en évidence la nécessité de promouvoir la visibilité du traducteur, nous croyons que la traduction engagée pour "promouvoir l'intercompréhension culturelle » (Gambier, 2005, p. 6) est un champ encore à définir. Un premier jalon cependant serait pour nous non seulement la critique de l'invisibilité du traducteur littéraire dans l'espace anglo-saxon (Venuti, 1995), mais bien plus l'affirmation radicale de la visibilité engagée, à la fois éthique et citoyenne, du traducteur dans les sociétés postcoloniales des grandes concentrations urbaines occidentales ou occidentalisées.

Lorsque Pym représente la responsabilité du « héraut-traducteur » par son pouvoir de se sacrifier, de s'effacer et proclame que "[l]'individu est libre de ne pas se faire valoir comme individu » (1997, p. 52), non seulement il souligne la liberté que possède le traducteur de choisir d'être invisible (plutôt que visible), mais il insiste en même temps sur l'absence du traducteur comme sujet. L' " agence » (Gouanvic, 2001) du traducteur est en quelque sorte subordonnée à un devoir d'effacement préalable : un bon traducteur est un traducteur qui sacrifie son individualité. Évoquant l'histoire de Sperthias et Boulius, que Plutarque a rapportée d’Hérodote, Pym en tire la leçon suivante :

\begin{abstract}
En fait, la seule reconnaissance de l'individu dans cette histoire semble être celle qui s’inscrit dans le mot «volontairement », répété par Plutarque-Amyot à deux reprises. Sperthias et Boulius choisissent de se faire tuer. Leur individualité est celle d’une subjectivité capable de calculer son propre anéantissement, de se sacrifier au bonheur abstrait de cette trinité congruente «pays, loi, hommes ». De la même façon, certains traducteurs choisissent "volontairement » de se passer d'un nom propre (le traducteur traduisant ne peux pas dire «je »), de se sacrifier symboliquement devant les intérêts d'un texte dit source. [...] On choisit d'être traducteur professionnel. (1997, pp. 51-52)
\end{abstract}

\footnotetext{
${ }^{8}$ Il est bien évident que nous référons dans cet article au traducteur dans son acception générique, à la fois féminine et masculine.
} 
Or, comment peut-on être responsables dans l'effacement ? Comment le traducteur peut-il espérer remplir son mandat de médiateur interculturel en ayant sa propre invisibilité pour condition? Pour Pym, c'est la conséquence du choix que fait l'individu d'être traducteur professionnel : atteindre « un degré d'impersonnalité » (p. 70).

Plus loin cependant, il ajoute que dans la relation de pouvoir entre le client et le traducteur, on peut voir celle qui existe entre le maître (le traducteur sans client) et l'esclave (le traducteur-machine) (p. 80), la responsabilité du traducteur se situant entre ces deux extrêmes. Ce qui le conduit à tirer le principe suivant : " moins le traducteur se trouve en position d'infériorité sociale, plus il est responsable de ses choix » (id.). Ce qui signifie donc que le traducteur doit être visible pour être responsable et libre de choisir.

Mais Pym distingue l'espace du traducteur et celui de la traduction, de même qu'il distingue l'individu et le traducteur. Si le premier " est théoriquement libre de faire tout ce qui n'est pas interdit ", le second n'a de liberté que dans « la capacité d'agir pour le compte d'un autre ». " Autrement dit, le traducteur est libre d'agir uniquement en tant que traducteur. Jamais comme individu » (p. 81). Cette schizophrénie qui consiste à séparer l'individu du traducteur viserait donc à distinguer le professionnel - qui fait abstraction de son individualité - de celui qui ne l'est pas. C'est comme si la caractéristique du professionnel est, d'un côté, de mettre en évidence les aspects extérieurs du traducteur qui servent à donner confiance aux clients (image, confiance, autorité, respect) (p. 71) alors que, d'un autre côté, il dissimule la dimension intérieure de l'individu habité par le seul « doute » face aux alternatives. L'invisibilité ne serait donc que celle de l'individu, le traducteur professionnel étant, quant à lui, pourvu d'une apparence qui le rend visible. Qu'en est-il donc de l'invisibilité du traducteur?

Quand bien même l'invisibilité ne serait que partielle, la tendance semble pourtant aller dans ce sens. Bien que Pym ironise sur le traducteur qui s'efface par souci de professionnalisme, ${ }^{9}$ il reste qu'il n'y a, pour lui, d'existence traductionnelle que dans le silence de son individualité :

\footnotetext{
${ }^{9}$ « Le traducteur qui ne fait que traduire, qui n'a pas de voix en dehors de la traduction, est certainement digne de confiance. Si vous voulez paraître fidèle, gardez le silence, ne théorisez pas, n'exprimez pas vos doutes, surtout en dehors des cercles des traducteurs. Telle serait la lâcheté secrète du masque professionnel » (1997, p. 82).
} 
Donner au traducteur une voix indépendante, c’est en fait valoriser un mode de causalité qui n'est plus celle de la forme traductionnelle (p. 93).

Et pourtant, Pym affirme qu’il est « largement d'accord avec la prise de position de Venuti » (en faveur de la manifestation du traducteur) (p. 95), même s'il reste sceptique à l'égard des moyens qu'il recommande (p. 97). En effet, alors qu'il comprend la nécessité de pratiquer une traduction résistante, il reproche cependant à Venuti non seulement de ne pas reconnaître l'importance des critères commerciaux, mais de vouloir en bénéficier tout en même temps (p. 96). Une telle liberté de traduire, même si on l'accuse d' " académisme subventionné », est une farouche opposition au mercantilisme qui tente de la réduire en esclavage. C'est que l'affirmation de la voix du traducteur, de son autonomie dans la causalité et de sa résistance aux «mouvements ethnocentriques et impérialistes qui sont forcément impliqués dans tout acte de traduction » (Venuti, 1991, p. 127) sont autant de signes annonçant l'avènement prochain de la souveraineté du traducteur.

Ni mercenaire, ni objet sacrificiel de l'exigence commerciale, le traducteur est bien plutôt cet agent de médiation « transculturel » (Nouss, 1997, p. 262) qui, prenant acte de ses responsabilités envers toutes les cultures auxquelles il appartient, prend la liberté de traduire en affirmant la cohésion entre son individualité et sa fonction traductive. Émancipé de la schizophrénie qui le partageait entre une intériorité et une extériorité supposées, le traducteur admet qu’il « faut plus que la traduction » (Pym, 1997, p. 137), qu’il faut s’engager pour se libérer des contraintes idéologiques et matérielles et se consacrer au bien-être des plus faibles (p. 126).

Du traducteur professionnel, l’on passe au traducteur engagé. Le critère essentiel n'étant plus de « coopérer ${ }^{10}$ avec le client pour amortir les investissements de temps et d'argent de part et d'autre, mais de pousser jusqu'au bout le rôle de « conseiller en communication » ou d' « expert en relations interculturelles » (p. 80). Le traducteur est responsable du fait qu'il a conscience de la distance qui sépare les cultures qui l'habitent en même temps. En ce sens, nous rejoignons complètement Pym lorsqu'il affirme que :

\footnotetext{
${ }^{10}$ « La coopération » est le titre du cinquième et avant-dernier chapitre du livre de Pym.
} 
Devenir traducteur, c'est d'abord voyager. C'est seulement ensuite qu'on atteint la condition de Blendling. Il faut d'abord que l'effort de l'individu soit investi dans le déplacement, non seulement pour réaliser un voyage matériel mais aussi pour traverser toutes les distances culturelles qui résultent du déplacement matériel, en premier lieu par l'apprentissage des langues. Est-ce qu'on naît traducteur? Est-ce que le traducteur est natif ou naturel ? C'est plutôt en voyageant vers d'autres cultures, en s'engageant dans les déplacements culturels, qu'on devient traducteur, genre particulier de Blendling, genre particulier de héraut. (p. 63)

«L'engagement dans les déplacements culturels », c’est aussi l'engagement dans la prise de conscience qui l'accompagne, l’éveil aux autres visions du monde qui en découle. Engagement dans la résistance à ce qui cherche à imposer l'illusion d'une nouvelle langue universelle, d'un seul moyen d'échange possible : le commerce. Car résister, ce n’est pas - comme on voudrait le faire croire par ailleurs ${ }^{11}$ - « arrêter le train du progrès ", ou s’opposer à l'irrésistible marche en avant de la modernité ou encore vouloir entraver de manière donquichottesque ce que l'on a décrit comme « la fin de l'histoire ». Résister, c’est opérer une transformation, pour le traducteur comme pour tout autre, qui le conduira du statut de pourvoyeur de service (rouage de l'économie) à celui de citoyen (électron libre) et de l'individu (membre d'un système) au sujet (fondateur de son autonomie). ${ }^{12}$

Mais résister ne peut se faire dans l'anonymat, au risque d’être confondu avec le terrorisme. C'est pourquoi, le traducteur ne peut s'en remettre au seul « masque professionnel » (p. 82) pour être visible et agir de façon responsable sous le regard de ses semblables, mais il doit nécessairement donner à voir son vrai visage. Nous dirons en ce sens, avec Lévinas, que «le Visage [...] est le commencement de l’intelligibilité » (Lévinas, 1991, p. 121).

Le traducteur est celui qui, tout en cherchant le visage de l'autre pour le traduire et « répondre de lui », doit tout à la fois s'en donner un dans l'acte même du traduire. C'est dans la mesure où le traducteur prend la responsabilité citoyenne de se manifester dans son écriture traductive que l'action qu'il entreprend, de rapprocher les visages et les

${ }^{11}$ C'est Anthony Pym lui-même qui a répondu à une question que nous lui avions posée en utilisant cet argument. Congrès CATS/ACT 2003 à Halifax, Nouvelle Écosse.

12 Je dois cette distinction entre l'individu et le sujet à Alexis Nouss. 
voix, devient juste. Le « traducteur-citoyen » est donc celui dont la tâche, au-delà de la seule comparaison des langues et des cultures, consiste à s'engager - à visage découvert - à porter la responsabilité de la cité (aujourd'hui planétaire), à voir dans les textes qu'il traduit les visages de celles et ceux qui n'ont pas de voix et à se soucier de les donner à voir et entendre au monde :

La rencontre d'Autrui est d'emblée ma responsabilité pour lui. [...] Parlons d'une prise sur soi du destin d'autrui. C'est cela la vision du Visage, et cela s'applique au premier venu. [...] Les hommes, les incomparables, ne doivent-ils pas être comparés ? À la prise sur soi du destin de l'autre est donc antérieure ici la justice. Je dois porter jugement là où je devais d'abord prendre des responsabilités. [...] Mais c'est à partir du Visage, à partir de la responsabilité pour autrui, qu'apparaît la justice, qui comporte jugement et comparaison, comparaison de ce qui est en principe incomparable, car chaque être est unique; tout autrui est unique. (Lévinas, 1991, pp. 121-122)

Pour le traducteur-citoyen, c'est non seulement que chaque texte est un être unique incomparable à un autre, mais c'est également l'acte même du traduire qui l'est. La visibilité du traducteur, comme toute attitude éthique, est un état qui ne peut se satisfaire de la « temporarité », mais doit nécessairement s'inscrire dans la continuité, la permanence. Résister, c'est aussi résister à la tentation de renoncer, résister à l'attrait d'une justice éphémère, d’une conscience étroitement locale :

L'heure de la Justice, de la comparaison des incomparables se «rassemblant» en espèces et genre humains. Et l'heure des institutions habilitées à juger et l'heure des États où les institutions se consolident et l'heure de la Loi universelle qui est toujours la dura lex et l'heure des citoyens égaux devant la loi. (p. 259)

Le droit du traducteur à s'engager pour la justice en appelle donc à la reconnaissance de sa capacité à la pratiquer. Cependant, l'un des défis auxquels il fait face aujourd'hui tient dans la place qu'il a au regard du droit de l'auteur qui l'administre. Quelle éthique pour un traducteur dont les prérogatives juridiques le confinent à la subordination à l'auteur de l'original ? Le droit de l'auteur ne peut plus être sourd à la revendication d'équité alors que les pays en développement sont invités à rejoindre l’OMC, nouvelle dura lex de ce millénaire.

Si l'on peut voir dans ce qui précède une revendication pour l'avènement du traducteur-citoyen, il s'en suit que sa tâche traductive actuelle, la traduction elle-même - ou une certaine pratique de celle-ci 
dans le cadre plus large d'une société démocratique qui a mieux intériorisé ses différences et ses richesses ainsi que dans celui d'un monde ressaisi par-delà les antagonismes civilisationnels d'un Huntington - est loin d'avoir été définie de manière irrévocable. En effet, la traduction se joint désormais au nombre des éléments sensibles qui permettent de mesurer l'étendue de la pratique démocratique. La traduction citoyenne est en quelque sorte le vecteur de transparence culturelle que favorise une société démocratique entre les différentes visions du monde que véhiculent ses acteurs. Le caractère métaphorique, au sens traditionnel, de ce type de traduction est donc pour le moins sujet à caution dans la mesure où même si l'on conçoit la traduction au-delà de son acception strictement linguistique, il reste qu'elle possède toutes les assises d'enjeux sociopolitiques réels.

\section{Conclusion}

Alors que cet article est intitulé « la traduction citoyenne n'est pas une métaphore ", nous avons cependant tenté de démontrer le contraire. D’une part, la traduction citoyenne est bien une métaphore au sens où nous assumons l'élargissement du champ de la traductologie à ce qui déborde le transfert linguistique et que, d'autre part, la traduction métaphorique est à la fois légitime, par l'urgence sociale qui l'appelle et l'interpelle au sein des sociétés démocratiques et hypermétissées d'aujourd'hui, et paradoxalement bien réelle puisque porteuse d’intercompréhension, de rapprochement et de coopération.

Qu'est-ce à dire ? Nous avions en fait deux objectifs. Le premier consistait à signifier, au premier abord et selon la compréhension courante, que la traduction qui nous intéresse est bien réelle et foncièrement légitime: en ce sens, elle n'est pas qu'une métaphore. Le second objectif, en revanche, visait à faire l'expérience d'une resémantisation de la traduction comme métaphore au point d'en déconstruire le sens premier et de la prendre à contre-courant, comme un des lieux de prédilection pour l'engagement social. La traduction comme métaphore non seulement ne s'embarrasse pas d'opérer et d'agir dans le monde avec des prétentions réparatrices, mais elle est devenue une nécessité à la fois sociale et politique dans la société traversée de tensions et de questionnements identitaires qu'est l'Occident contemporain. La pacification des espaces de citoyenneté locale par l'effort traductif des représentations et des perceptions est le seul espoir d'un probable vivre-ensemble dans nos sociétés respectives et peut-être plus largement dans le monde. 
La puissante doxa technologique du troisième millénaire n'est autre que la traduction forcée de tout ce qui paraît faire silence, qu'on ignore ou qu'on n'entend pas, même au plus fort de sa revendication à l'ère de l'information. Dominer ne consiste plus à museler l'autre, mais bien au contraire à le faire parler quand on le décide pour lui, voire à parler directement en son nom. Comment s'en libérer ? La «traduction citoyenne " que nous proposons cherche cette libération, mais encore faut-il que la traductologie libère son objet des carcans définitoires que d'aucuns lui imposent.

\section{Université d'Ottawa}

\section{Références}

BARSKY, Robert (1994). Constructing a Productive Other: Discourse Theory and the Refugee Hearing. Amsterdam, John Benjamins.

- (2000). Arguing and Justifying: Assessing the Convention Refugees' Choice of Moment, Motive and Host Country. Burlington (USA), Ashgate Publishing.

BASSNETT, Susan et André LEFEVERE (1990). Translation, History and Culture. London, Pinter.

BOZEMAN, Adda (1971). The Future of Law in a Multicultural World. Princeton, Princeton University Press.

CHEYFITZ, Eric (1991). The Poetics of Imperialism, Translation and Colonization from the Tempest to Tarzan. Philadelphia, University of Pennsylvania Press.

CLIFFORD, James et G. E. MARCUS (eds) (1986). Writing Culture. The Poetics and Politics of Ethnography. Berkeley, University of California Press.

COPELAND, Rita (1991). Rhetoric, Hermeneutics, and Translation in the Middle Ages: Academic Traditions and Vernacular Texts. Cambridge, Cambridge University Press.

GAMBIER, Yves (2005). "Pertinence sociale de la traductologie ? ». Meta, 50, 4, http://www.erudit.org/livre/meta/2005/000206co.pdf 
GOUANVIC, Jean-Marc (2001). "Ethos, éthique et traduction : vers une communauté de destin dans les cultures ». TTR, XIV, 2, pp. 31-47.

GUSDORF, Georges (1986). "Connaissance Interdisciplinaire ». Encyclopaedia Universalis. Paris, Encyclopaedia Universalis France S.A, vol. VIII, pp. 1086-1090.

JAKOBSON, Roman (1963). Essais de linguistique générale. Vol. 1, trad. Nicolas Ruwet. Paris, Éditions de Minuit.

LAPLANTINE, François (1995). «L'ethnologue, le traducteur et l'écrivain ». Meta, 40, 3, pp. 497-507.

LÉVINAS, Emmanuel (1991). Entre nous. Essais sur le penser l'autre. Paris, Grasset, coll. « Figures ».

LIENHARDT, Godfrey (1967). "Modes of Thought ", dans Evans-Pritchard, Firth, Leach, Peristiany, Layard, Gluckman, Fortes and Lienhardt, The Institutions of Primitive Society. Oxford, Basil Blackwell, pp. 95-107.

MORIN, Edgar (1990). "Articuler les disciplines », http://wwwmcxapc.org/docs/conseilscient/morin1.html

— (1999). La tête bien faite. Paris, Éditions du Seuil.

NIRANJANA, Tejawsini (1992). Siting Translation. History, Post-Structuralism, and the Colonial Context. Berkeley, Los Angeles and Oxford, University of California Press.

NOUSS, Alexis (1995). «La traduction comme OVNI ». Meta, 40, 3, pp. 335-342.

- (1997). « Compte rendu de lecture, Pour une éthique du traducteur d'Anthony Pym ». TTR, XI, 1, pp. 258-263.

OUSTINOFF, Michaël (2003). La traduction. Paris, PUF, coll. «Que sais-je ? » $n^{\circ} 3688$.

PEETERS, Jean (1999). La médiation de l'Étranger. Une sociolinguistique de la traduction. Arras, Artois Presses Université. 
PYM, Anthony (1997). Pour une éthique du traducteur. Arras, Artois Presses Université et Presses de l’Université d'Ottawa.

RAFAEL, Vincent (1988). Contracting Colonialism. Translation and Christian Conversion in Tagalog Society under early Spanish Rule. Ithaca, N.Y., Cornell University Press.

RICOEUR, Paul (1986). Du texte à l'action. Paris, Éditions du Seuil, « coll. Points ».

SNELL-HORNBY, Mary (1990). « Linguistic Transcoding or Cultural Transfer? A Critique of Translation Theory in Germany ». Translation, History and Culture, Susan Bassnett and André Lefevere (Eds). London, Pinter, pp. 79-86.

STEINER, George (1998). Après Babel. Une Poétique du dire et de la traduction. Paris, Albin Michel.

VALERO-GARCES, Carmen (1995). « Modes of Translating Culture: Ethnography and Translation ». Meta, 11, 4, pp. 556-563.

VENUTI, Lawrence (1991). «Genealogies of Translation Theory: Schleiermacher ». TTR, IV, 2, pp. 125-150.

- (1998). The Scandals of Translation. Towards an Ethics of Difference. London and New York, Routledge.

WOLF, Michaela (2002). "Culture as Translation - and Beyond Ethnographic Models of Representation in Translation Studies », dans Theo Hermans (dir.), Crosscultural Transgressions. Manchester, St-Jerome, pp. 180-191.

RÉSUMÉ : La traduction citoyenne n'est pas une métaphore Après une mise en question de l'étroitesse de la définition de la traduction comme une opération de transfert exclusivement interlinguistique, cet article explore la possibilité d'élargir les frontières définitoires, établies au sein de la discipline traductologique, par un retour sur la valeur, pour le moins dépréciée, de la traduction métaphorique. La tentative consiste à faire, d'une part, un détour par l'éthique du traducteur qui impose sa visibilité et, par conséquent, sa participation citoyenne dans l'effort de rapprochement et d'intercompréhension des différences, et, d'autre part, une 
resémantisation du caractère métaphorique de la traduction dès lors qu'elle est porteuse d'enjeux aussi réels et circonstanciés que ceux, par exemple, de la difficile intégration des musulmans occidentaux et de l'expression de leur religiosité dans les sociétés laïques. En somme, la traduction métaphorique ainsi comprise nous conduit à considérer le nouveau concept de « traduction citoyenne ».

ABSTRACT: Civic Translation Is Not a Metaphor - After questioning the current definition of translation, which is narrowly restricted to the inter-linguistic transfer of texts, this article explores the possibility of expanding the definitional boundaries set by translation studies by returning to the undervalued notion of metaphorical translation. This research will be conducted from two inter-related vantage points: firstly, the translator's ethics, his or her visibility and the resulting acknowledgement of his or her civic participation in the meeting and mutual comprehension of cultural difference; and secondly, the re-valuing of translation in its broader metaphorical sense, as a notion bearing upon such socially relevant and complex issues as, for example, the difficult integration of Western Muslims, as well as the expression of their religiosity in secular societies. Understood in this manner, metaphorical translation may help us to consider the new concept of "civic translation."

Mots-clés : métaphore, définition de la traduction, citoyenneté, visibilité, démocratie.

Keywords: metaphor, definition of translation, citizenship, visibility, democracy.

Salah Basalamah: École de traduction et d'interprétation Université d'Ottawa, 70 Laurier Est, bur. 529

Ottawa, ON - K1N 6N5

Courriel : salah.basalamah@uottawa.ca 